\title{
La morbilidad reemplaza a la mortalidad: un dilema ético en el cuidado de los prematuros en los límites de la viabilidad
}

\author{
Morbidity replaces mortality: an ethical dilemma in the care of premature babies \\ at the limits of viability
}

Los cuidados médicos de pacientes críticamente enfermos se han vuelto progresivamente más complejos y costosos en todas las áreas de la medicina. Varios de esos pacientes logran sobrevivir gracias a una actitud loable que lleva a realizar múltiples esfuerzos e involucra a muchos profesionales de la salud que trabajan con gran dedicación y compromiso.

Sin embargo, en ocasiones no infrecuentes esto se alcanza sin reparar ni en la evolución posterior, ni en la complejidad, ni en los costos, ni tampoco en los deseos del paciente y/o de su familia. Es por lo tanto necesario que para que el fin de esa difícil tarea sea apropiado se realice una reflexión muy profunda de varios aspectos que entran en diversos terrenos de la medicina o son afines a ella tales como el de la ética, el de la equidad en el cuidado de la salud, el de la sustentabilidad del sistema y en la definición de cuáles son las metas de la medicina.

Un ejemplo palpable de esta situación se observa en las unidades de cuidado intensivo neonatal (UCIN) producto de los inmensos y vertiginosos avances ocurridos en la neonatología, uno de los más notables en medicina. Los progresos trajeron innumerables beneficios pero que al mismo tiempo se acompañaron de diversos y nuevos problemas, principalmente en el campo de la ética.

El punto más crítico está centrado en la atención de los prematuros extremadamente pequeños (menores de 26 semanas o de $800 \mathrm{~g}$ ). En este grupo, los de mayor riesgo son los que se encuentran en los límites inferiores de la viabilidad, algo que se fue modificando sustancialmente en los últimos 30 años. Hasta la década de 1970 el límite se consideraba en la semana 28 , años después en la semana 26 y a partir de la década de 1990, en la semana 24. Actualmente, la mayoría de las Sociedades Científicas sigue considerando ese criterio aunque en algunos países europeos se fija en 25 o 26 semanas y en otros lugares en 23. Asimismo, las recomendaciones de las guías consideran apropiado no iniciar reanimación en recién nacidos (RN) menores de 23 semanas. No obstante, en varias unidades neonatales, en especial de los EE.UU., esa práctica se realiza incluso en prematuros de 21 y 22 semanas.

En los últimos 20 años se observa un significativo aumento en la supervivencia de $\mathrm{RN}$ a las 24 y 25 semanas, es decir en los límites señalados. Si bien este logro tiene sin dudas repercusiones bene- ficiosas, al mismo tiempo va generando algo que cada vez preocupa más, el reemplazo de la mortalidad por morbilidad. Esto repercute no solo en el período neonatal sino en especial en la enorme implicancia futura de las secuelas a largo plazo sobre el niño, la familia y la comunidad.

Para celebrar los 75 años de la Sociedad Argentina de Pediatría el Dr. Gianantonio, por entonces su presidente, organizó en 1986 un Simposio que denominó "La nueva morbilidad", un enfoque sumamente llamativo y no abarcado en la Pediatría hasta ese momento. En el temario se incluyó la morbilidad alejada de los prematuros pequeños que en esa época recién comenzaban lentamente a tener una menor mortalidad. Con su admirable clarividencia, Gianantonio intuyó los problemas que se iban a producir y que hoy tenemos en una proporción creciente.

Varias razones explican la mayor supervivencia, en especial los marcados avances en los conocimientos y el gran desarrollo tecnológico. Eso ocasionó que las UCIN comenzaran a poblarse de prematuros muy pequeños que requerían cuidados progresivamente más complejos y permanecían meses internados. Este aspecto, sumado a la continua y perjudicial mercantilización de la medicina, donde cuantos más tratamientos y estudios se realicen, mayores serán las ganancias, motivó que la asistencia de los prematuros fuera algo muy rentable para algunas instituciones. Con relación a este aspecto, Willian Silverman, considerado por sus méritos excepcionales el padre de la neonatología moderna, advirtió reiteradamente sobre los graves problemas que ocasionaba en EE.UU. lo que él denominó, la "industria del cuidado neonatal intensivo". En un comentario que denominó "Compasión u oportunismo" (Pediatrics 2004;113;402) señaló entre otras cosas que el costo en el cuidado intensivo de los prematuros en el año 2000 alcanzó la exorbitante cifra de 12000 millones de dólares, sin contar honorarios profesionales. En su opinión, las UCIN se habían convertido en "centros de ganancias" y era incrédulo suponer que este aspecto no influía en las decisiones médicas de tratamientos de rescate no justificados en prematuros extremadamente pequeños.

Es innegable que los cuidados médicos de estos pacientes caminan, en forma cada vez más pronunciada, en la delicada y borrosa línea que separa 
lo que se puede hacer de lo que se debe hacer. En alguna medida, esto podría relacionarse con la incertidumbre acerca del futuro de los bebés que presentan trastornos graves y que requieren todos los elementos terapéuticos disponibles para mantenerlos con vida. Sin duda, frecuentemente es difícil definir qué niños sobrevivirán sin trastornos, o con algunos leves, y cuáles, en cambio, tendrán secuelas graves e irreparables de por vida.

No obstante, de ninguna forma este aspecto puede justificar que los neonatólogos y profesionales auxiliares eviten analizar sistemáticamente la posible futilidad de las conductas que se toman a fin de no cometer acciones éticamente objetables y entrar en el lamentable terreno del encarnizamiento terapéutico.

La magnitud de los problemas que pueden presentar a largo plazo los prematuros extremos se refleja en el estudio de Marlow y col. (NEJM 2005 ; 352:9-17) que evaluaron hasta los 6 años una extensa cohorte de RN entre las 22 y 25 semanas y hallaron algo especialmente dramático: la tasa de supervivencia sin ninguna discapacidad a los 6 años fue de $0 \%$ en los nacidos a las 22 semanas, $1 \%$ a las 23 semanas, $3 \%$ a las 24 semanas y $8 \%$ a las 25 semanas.

Si bien, tanto las actitudes o estrategias intervencionistas como las abstencionistas pueden ser controvertidas, en general se observan al menos dos conductas bien diferentes en los cuidados que se brindan, lo cual muestra una distinta forma de pensar y por lo tanto de actuar.

Hay grupos que reaniman a prematuros sumamente pequeños aun cuando los signos vitales sean débiles y aplican todas las medidas posibles de tratamiento intensivo aunque la probabilidad de sobrevivir sea muy escasa. Se trata de mantener la vida "por encima de todo" y por lo tanto no se repara en el pronóstico a largo plazo ni en el impacto futuro de una discapacidad grave. Tampoco esta estrategia suele tener en cuenta los deseos de los padres ya que no les da lugar a una participación más activa.

Una conducta intermedia tiene en cuenta las dificultades de poder definir acertadamente en las etapas tempranas cuál será la evolución inmediata y el pronóstico alejado. Por lo tanto, ante la incertidumbre, inicialmente se aplican medidas de rescate y tratamientos intensivos con decisiones individualizadas que se van revisando hasta tanto se pueda tener mayor seguridad en la decisión de continuar con el tratamiento o interrumpir el soporte vital.

Los padres están informados de los riesgos que existen y de que las medidas terapéuticas pueden ser transitorias si los cuidados médicos no modifican la situación. Cada una de las posibilidades se conversa con ellos en forma honesta, con un lenguaje comprensible y realista, de manera que tengan una adecuada información y puedan comprender que el mayor interés de los médicos y del personal de enfermería es la salud de su hijo. De esa manera los padres irán adquiriendo una mayor confianza en los cuidadores de su bebé y, a mi juicio, esto constituye uno de los elementos esenciales de esta compleja situación. La confianza es un aspecto básico e imprescindible en toda relación humana.

Esta conducta, a mi criterio la más razonable, se basa en el principio de autonomía, que implica el derecho de los padres de conocer y participar en los aspectos concernientes a la vida de sus hijos gravemente afectados. No obstante, es una tarea sumamente difícil, que exige de los profesionales una extrema dedicación y convencimiento de que están haciendo lo más apropiado para el niño y su familia.

Sin embargo, surge una pregunta que está en el centro del dilema ético ¿Cuál es el cuidado apropiado? Es difícil responderla con certeza ya que como he señalado hay muchos factores que "rodean" al médico cuando debe tomar decisiones y suelen influenciarlo en su conducta. A mi entender, la mejor respuesta es aquella que define al cuidado apropiado cuando se basa en decisiones compartidas destinadas a la mejor opción para el paciente y su familia. Esto se logra al trabajar en un grupo donde las conductas a tomar sean conversadas en forma abierta entre los profesionales médicos, el personal de enfermería, cuyas valiosas opiniones no pueden estar ajenas a las deliberaciones, y la participación de los padres. Cuando las dudas persisten, en general estos grupos recurren a los comités de ética institucionales, que es una instancia muy enriquecedora y suele ser de una ayuda sumamente importante.

Finalmente, debemos aceptar que el abordaje desde la bioética en la asistencia de estos $\mathrm{RN}$ es sumamente difícil y representa una enorme tensión emocional para los profesionales en la búsqueda del equilibrio entre las conductas proporcionadas y desproporcionadas. Asimismo, son evidentes nuestras limitaciones para brindar un cuidado que no solo esté destinado a lograr que estos niños sobrevivan, sino también a aplicar en las unidades neonatales medidas eficaces que protejan su tan vulnerable desarrollo neurológico y cognitivo en un intento de mejorar el pronóstico a largo plazo.

$$
\text { José M. Ceriani Cernadas }
$$
Editor

http://dx.doi.org/10.5546/aap.2012.98 